\title{
A non-invasive capacitive sensor strip for aerodynamic pressure measurement
}

\author{
M. Zagnoni ${ }^{\text {a* }}$, A. Golfarelli ${ }^{\mathrm{a}}$, S. Callegari ${ }^{\mathrm{a}}$, A. Talamelli ${ }^{\mathrm{b}}$, \\ V. Bonora ${ }^{\text {c }}$, E. Sangiorgi ${ }^{\mathrm{a}}$, M. Tartagni ${ }^{\mathrm{a}}$ \\ a ARCES, University of Bologna, Via Fontanelle 40, 47100 Forlí, Italy \\ b DIEM, University of Bologna, Via Fontanelle 40, 47100 Forli, Italy \\ c DICASM, University of Bologna, Via Fontanelle 40, 47100 Forli, Italy
}

Received 13 September 2004; received in revised form 15 March 2005; accepted 18 March 2005 Available online 23 May 2005

\begin{abstract}
This paper presents a capacitive pressure sensor strip implemented in general purpose printed circuit board (PCB) technology based on a thin 3D structure composed of polyimide, woven glass reinforced epoxy resin (FR4) and metal layers. Multiphysics finite elements method (FEM) simulations have been performed over the proposed structure in order to develop a time-dependent electrical and mechanical model that can be easily used to tailor the characteristics to the application. The device targets a wide class of fluid dynamics applications, being non-invasive, comformable and smart for placement. The device simulations are herein validated by experimental wind tunnel measurements and compared with figures obtained on a wing profile by conventional electromechanical pressure transducers. This approach is one of the first example of fully embedding and electronically controlled fluid flow monitoring apparatus that could be used in replacement of state of the art mechanical systems.
\end{abstract}

(C) 2005 Elsevier B.V. All rights reserved.

PACS: $81.05 . \mathrm{Lg}$; 83.60.Bc; 84.37.+q; 89.20.-a

Keywords: FEM; Polyimide; Capacitive sensors; Pressure sensors; Fluid dynamics

\section{Introduction}

Pressure measurements are of great importance in almost all field of engineering and industrial application. The recent development of numerical codes to calculate fluid flow has not diminished the need of detailed space and time resolved measurements, both to provide boundary condition and to validate the results. Despite the large amount of literature on the subject [1], whenever sensors are used in situ to monitor pressures on large domains in highly unsteady flows, there are still problems in using classical techniques due, for instance, to the cost of each single transducer, their intrusiveness or their time response.

In transport related industry, sensors are essential for monitoring the fluid dynamic environment, required for instance

\footnotetext{
* Corresponding author. Tel.: +39 0543 786924; fax: +390543 786926 .

E-mail address: mzagnoni@deis.unibo.it (M. Zagnoni).
}

in aerospace, ground vehicle and nautical applications, and for the aerodynamic body optimization during the design phase. Although similar, the above mentioned applications are characterized by very different environments, requiring sensor features and specifications that are very different from each others. For instance, sensors for aircraft design need high accuracy and precision, working in ranges up to $2 \mathrm{kPa}$. Conversely, sensors for internal airflow for automotive applications require a reduced sensitivity with respect to the previous ones, operating in a range of pressure from 10 to about $30 \mathrm{kPa}$ and requiring fast dynamic response with regard to the fluid to be monitored. Finally, sensors for nautical applications must be able to resist to wet environments and they must detect pressure ranges up to $300 \mathrm{~Pa}$.

A common specification in most of the above applications is related to the large size of the surfaces that has to be monitored, leading to the use of a large number of sensors in order to achieve the required spatial resolution. In this scenario, a 
real-time pressure distribution represents an important mean for the analysis of the aerodynamic behavior of the body and for its correct trim.

Modern pressure sensors that are used in this environment are silicon based capacitive sensor [2-4]. Silicon sensors can reach high sensitivity and accuracy, however they scarcely address all the requirements of the above industrial applications, such as high robustness and low manufacturing costs. On the other hand, standard fluid dynamics techniques, as Pitot tube, Prandtl tube or other optic techniques [5,6], are not able to satisfy, in many cases, either the low invasiveness (due to the presence of the pressure tubes) or the required accuracy, reducing their range of applications.

\section{Aims and sensor structure}

In the last few years printed circuit board (PCB) technology has greatly improved, achieving the photolithographic resolutions of silicon planar technology in the earlier 1970s, thus allowing the design of low cost precision transducers [7,8]. Materials such as polyimides and polyesters are now available in thin films of tens of $\mu \mathrm{m}$, allowing PCB devices to be used other than connecting electronic components but also as means for mechanical transduction. By using PCB technology it is possible to build up devices achieving most of the specifications required by fluid dynamic applications at low cost. Herein the sensors structure is presented together with the simulation methodology by which these devices can be optimally designed for the targeted application. Furthermore, the use of PCB technology has advantages over other approaches: it allows to naturally host electronic sensing and signal processing components by means of smart packaging such as the chip on board (COB) technology.

The sensor presented in this paper is a capacitive differential pressure transducer built in PCB technology as shown in Fig. 1. The sensitive unit consists of a three layer structure in a stack (Fig. 2): a rigid copper-clad glass-fiber base, a rigid glass-fiber spacer and a $25 \mu \mathrm{m}$ thick deformable copperclad Kapton ${ }^{\circledR}$ polyimide layer. Layers are attached to each other by means of a $50 \mu \mathrm{m}$ thick biadhesive tape, patterned in the same shape as the spacing layer. The device length and width can be set according to the application: the measure-

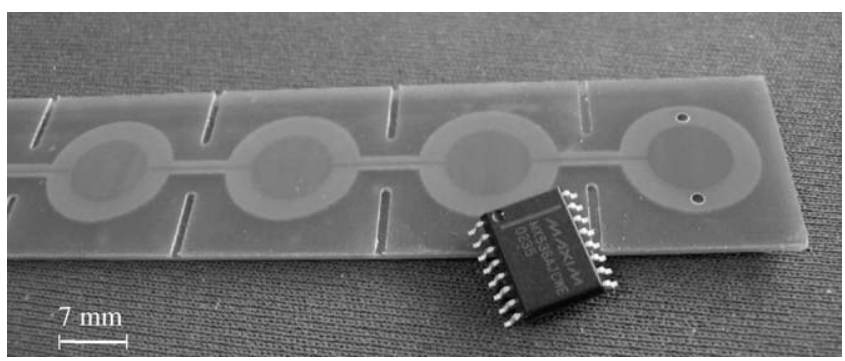

Fig. 1. PCB pressure sensor strip.

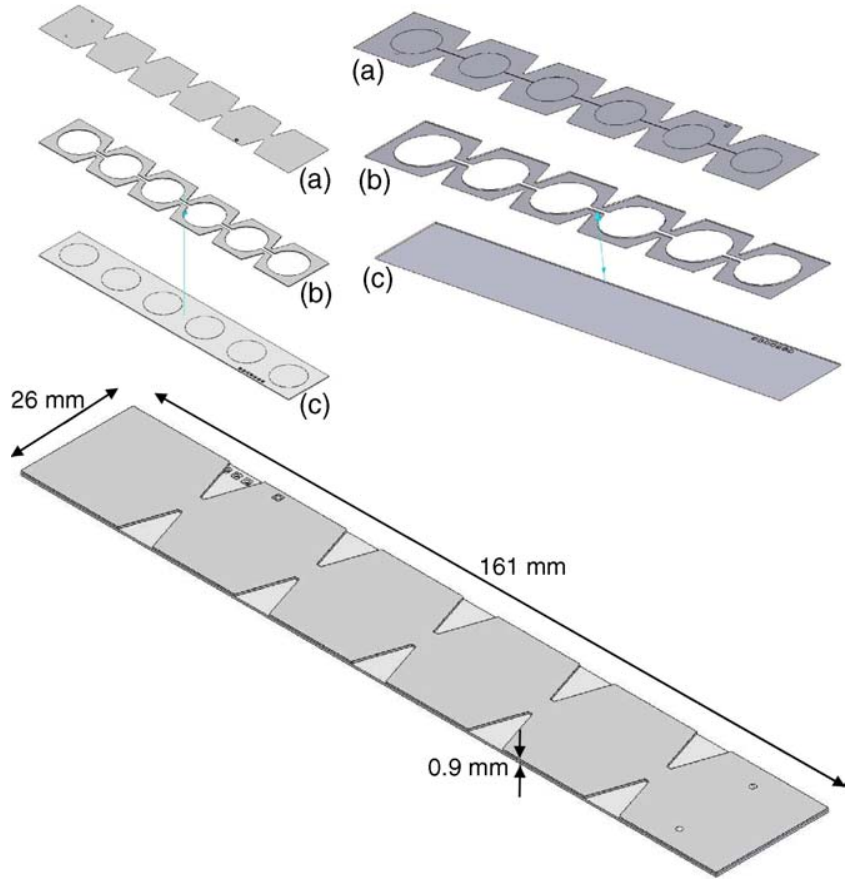

Fig. 2. PCB pressure sensor strip structure: (a) deformable copper-clad polyimide layer, (b) rigid glass-fiber spacer, (c) rigid copper-clad glass-fiber base. Exploded top view (up left side). Exploded bottom view (up right side). Assembled view (bottom).

ments and simulations of this paper are related to devices that are from 13 to $16 \mathrm{~cm}$ long and from 1.5 to $3 \mathrm{~cm}$ large. The total thickness is below $1 \mathrm{~mm}$. Each unit can be electronically readout in a multiplexed fashion in order to collect a set of surface pressure points, depending upon the application (Fig. 3). All chambers are connected by miniaturized pipes, patterned in the spacing layers, in order to share the same internal pressure. Small holes, drilled on the sides of

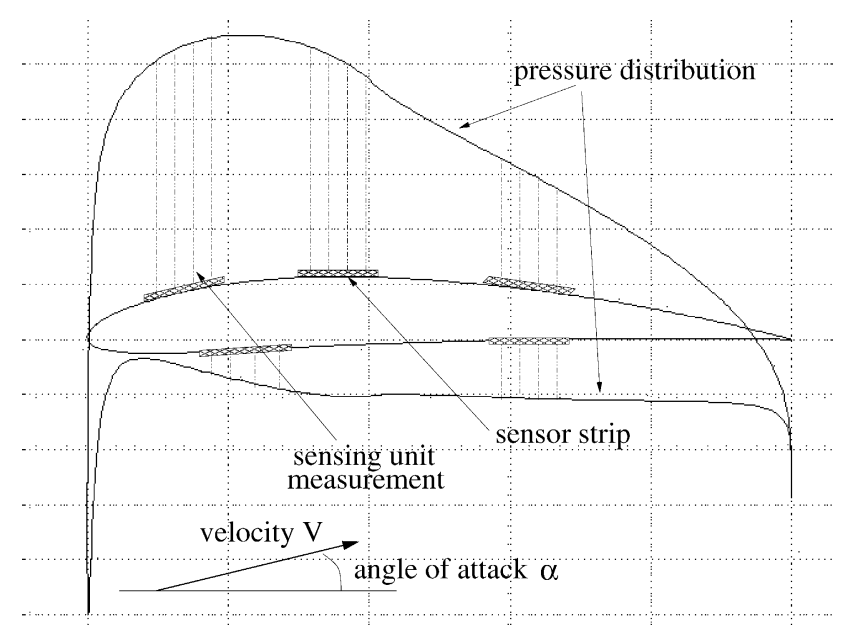

Fig. 3. Application example: monitoring pressure distribution over a wing profile. The pressure distribution over the profile depends by free stream velocity $V$ and angle of attack $\alpha$ : a changing in the $(\alpha, V)$ field leads to a different pressure pattern. 


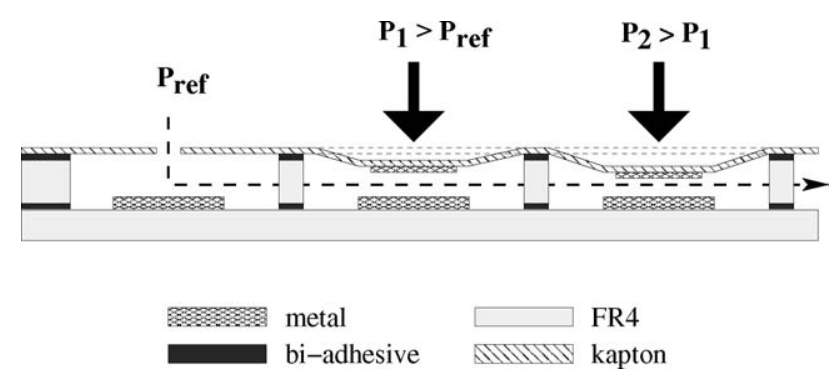

Fig. 4. PCB differential pressure sensor strip working principle. Membranes deflect upward or downward with respect to the gradient of pressure between outside and inside the chamber.

the first unit membrane or obtained in the spacing layer, act as a reference. As illustrated in Fig. 4, the membrane at each point of sensing deforms itself downward or upward with respect to the static pressure reference taken by means of the holes. Since the membrane area is usually much smaller than the aerodynamic surface to be monitored, the corresponding pressure distribution over the deformable film can be considered constant with a good approximation. The differential pressure sensors approach is very useful in aerospace environment: it overcomes altitude problems whereas absolute pressure sensors are affected by barometric pressure gradients. Membrane displacements due to the pressure induce variations of electrical capacitance between upper and lower conductive plates that can be easily readout by electronic circuitry. Capacitive sensors offer advantages as low power consumption, high sensitivity and low temperature dependence.

\section{Multiphysics simulations}

The sensor design is a fundamental task which involves understanding the range and sensitivity of the PCB units and to make comparisons to commonly used silicon MEMS structures. With the aid of FEM simulation, it is possible to describe most of the physical and structural sensor behavior in order to find optimum geometry, and to choose best materials to satisfy the specific application. Since the output of the sensor is a capacitive information, coupled electrical and mechanical simulation have to be taken into account. Fortunately, simple hand calculations show that, in contrast with typical MEMS structures, electrostatic force is negligible with respect to external forces inducing mechanical deformation: for a parallel circular plate capacitor with a radius of $1 \mathrm{~cm}$, the distance among armatures should be tens of $\mu \mathrm{m}$ for obtaining an electrostatic force, in air, equivalent to $5 \mathrm{~Pa}$ for an applied difference of potential of $5 \mathrm{~V}$. As a consequence, electrostatic simulations may follow mechanical ones without coupling. On the other hand, an important difficult issue is due to viscoelastic behavior of polymers. This problem is usually referred to as creep and its modeling is described in Section 3.3.

\subsection{Approximated reference model}

Modeling of plate deformations with respect to pressure should be carefully taken into account for design purposes. Whenever small mechanical perturbations are applied, classical mechanical theory of deformation can be used, where a linear stress-strain relationship for matters (Hooke law) is used. In this case, linear deformation of plates with respect to pressure is expected. However, whenever large deformations are applied, a specific theory has to be considered [9]. The increase of bending of a circular plate creates a strain in the middle plane that can not be neglected in cases where the deflections are no longer negligible with respect to the plate thickness. At the same time, large deflections in a plate cause supplementary stresses with respect to the conventional elastic theory and they must be taken into consideration in deriving the differential equations. This is a geometrical effect that causes non linearity between stress and deflection: the more a plate is stressed, the less it deflects. One should distinguish between two kinds of plates: thin plates with large deflections and thick plates. Sensors membrane, because of its thickness, is supposed to be described by the large deflections theory for thin plates with a good approximation. An useful relationship for an appropriate calculation of the deflections can be obtained by applying the energy method [9]. Considering a circular plate of radius $a$ be clamped at the edge and be subject to a uniformly distributed pressure $p$ and assuming the shape of the deflected surface represented by:

$w=w_{0}\left(1-\frac{r^{2}}{a^{2}}\right)^{2}$

where $r$ is the radius coordinate whose origin is set in the center of the membrane and $w_{0}$ the maximum deflection, the relationship of the displacement with respect to pressure is given by:

$w_{0}=\frac{p a^{2}}{64 D} \frac{1}{1+0.488\left(w_{0}^{2} / h^{2}\right)}$

where $D=\frac{E h^{3}}{12\left(1-v^{2}\right)}, E$ is the modulus of elasticity, $v$ is the Poisson ratio and $h$ is the plate thickness. Eq. (2) is typically used for $w_{0}$ greater than $0.5 \mathrm{~h}$ and it shows how the rigidity of the plate increases with respect the deflection, so that $w_{0}$ is no longer proportional with respect to the pressure, as in the elastic theory where $w_{0}=\frac{p a^{4}}{64 D}$. In the case of very thin plates, where $w_{0} \gg h$ a useful relationship is given by the following [10]:

$w_{0}=0.662 a \sqrt[3]{\frac{p a}{E h}}$.

This relationship shows that the deflection changes as the cube root of the intensity $p$. The above equations are a first useful tool for a rough estimation of the sensor dimensions. Assuming to build the sensor membrane with homogeneous materials and considering pressure values up to $2 \mathrm{kPa}$, one 
can describe the membrane deflections with Eqs. (2) and (3) for a plate thickness of tens of $\mu \mathrm{m}$ (i.e., as provided by PCB technology). Using the above expressions, it turns out that circular shaped membranes having diameters of about $1 \mathrm{~cm}$ can show deflections in the order of hundreds of microns in the given pressure ranges. This is precisely the amount of deflection needed for having the optimal electrical capacitance dynamic range. Unfortunately, this theory is very satisfactory in case of an homogeneous material, but not for composite laminates, where FEM non-linear algorithms need to be used to refine sensors design.

\subsection{FEM models}

Among the many possible FEM packages now available, Femlab [11] has been used as finite elements software. The simulation has been organized in the following mode: first, the sensor geometry is solved for the mechanical large deflection problems, static and time dependent, that gives as a result the membrane deflection, then the respective capacitance value is calculated. With this approach one can establish the sensor characteristic in absence of creep and add afterward the time dependent creep contribution as an error, calculated as a worst case. This procedure will be described in Section 3.3. The solution for the mechanical static large deflections problem is obtained solving the equilibrium equations for an axial symmetric problem, revealing that the creep behavior can not be neglected because of the stresses present in the membrane. Femlab solves for large deflections using a straindisplacement relation, known as Green or Green-Lagrange strains [12] and defined as:

$\epsilon_{\mathrm{GL}}=\frac{1}{2}\left(\frac{l^{2}-l_{0}^{2}}{l_{0}^{2}}\right)$,

where $\epsilon$ is the deformation, $l_{0}$ the initial length and $l$ the deformed length. This formula has been used in place of classical engineering strains, defined as $\epsilon_{\mathrm{E}}=\left(\frac{l-l_{0}}{l_{0}}\right)$. Furthermore Cauchy stresses, defined as $\sigma_{\mathrm{C}}=\frac{F}{A}$, are replaced by second Piola Kirchoff stress:

$\sigma_{\mathrm{PK}}=\frac{l_{0}}{l} \frac{F}{A}$,

where $\sigma$ is the stress, $F$ the force and $A$ the surface. This model has been used for the whole structure, even for the parts that are subjected to small deformation: this because the large deflections model is valid with a good approximation also for small displacement. By means of static simulations the stresses on the membranes have been calculated. As it will be better explained in the following paragraph, the values obtained by simulation indicate the necessity to enrich the model with viscoelastic behavior. To this aim, a time dependent large deflections FEM model has been made, using an exponential in time modulus of elasticity. As second step, for any deformed geometry obtained by mechanical FEM analysis, the Poisson equation $\left(\nabla^{2} V=-\frac{\delta}{\epsilon}\right)$ in the internal (a)

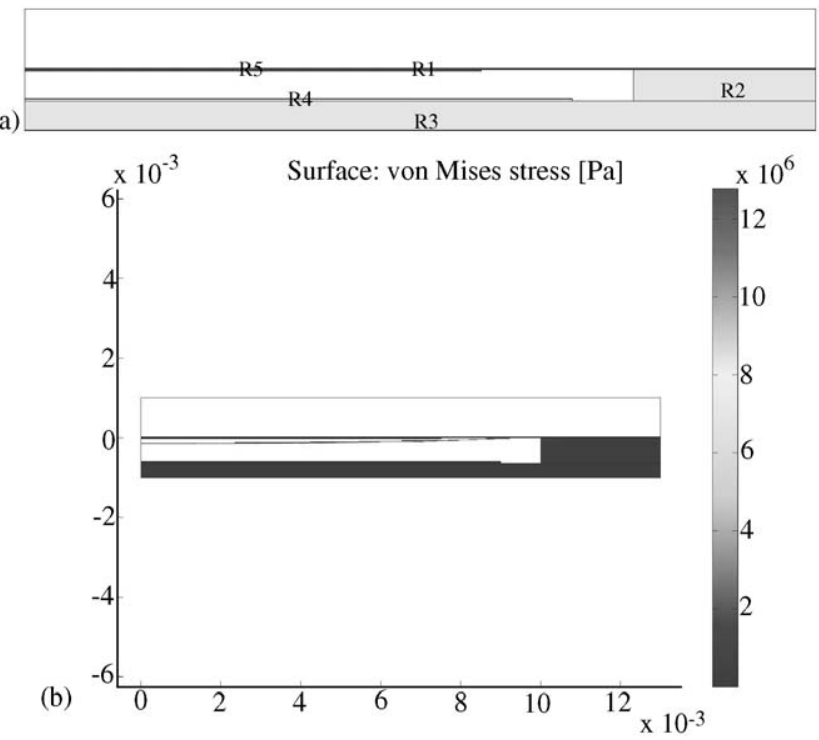

(c)

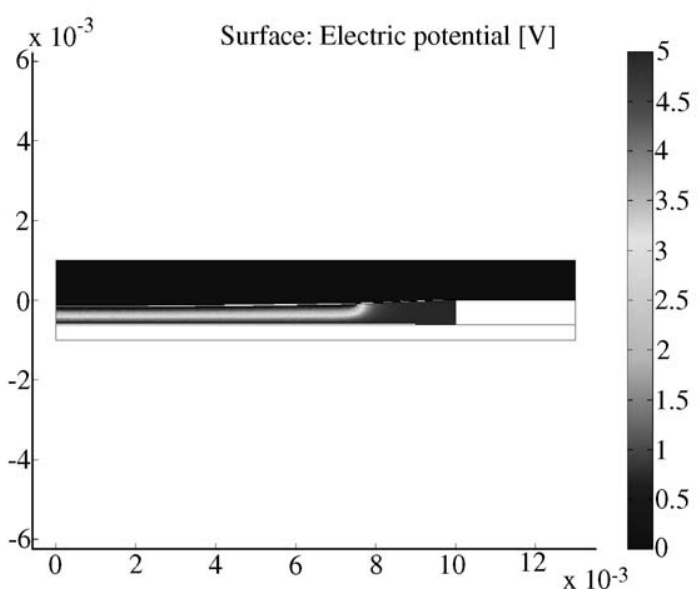

Fig. 5. FEM simulations graphical results: (a) sensitive unit geometry, (b) mechanical simulations: stress pattern, (c) electrostatic simulation: electric potential pattern.

chamber of the sensor is solved for the space charge density $\delta$ variable, where $V$ is the electrostatic potential and $\epsilon$ the permittivity. Integrating $\delta$ over the plate area of the sensor, the total capacitance $C$ is obtained as:

$C=\frac{Q}{V}=\frac{2 \pi}{V} \int_{0}^{R} \delta(r) r \mathrm{~d} r$

where $R$ is the radius of the electrode. To summarize, from this analysis the sensor membrane deflection are calculated as the response to the respective applied pressure distribution, finding out the capacitance variation, due to the geometrical changing (Fig. 5).

\subsection{Viscoelastic model}

Viscoelastic behavior of polymers has to be considered [13]. These properties depend upon their chemical structure and morphology, the size of the applied load and, crucially, temperature. An important implication of viscoelastic behav- 
iors is that the stress-strain characteristics can not be rigorously considered a static (i.e. memory-less, though nonlinear) relationship as depicted in Section 3.2. Conversely, the stress-strain characteristic exhibits behaviors that appear highly non linear, even for small deformations, and that, most important, depends on the derivatives of the stress and strain functions. This phenomenon is well evidenced by the analysis of a sample case: if a stress function step is applied to a sample of material subject to creep, a sudden elastic strain is followed by a viscous and time dependent strain with an increasing trend. Conversely, if a strain step is applied, the stress decreases as a monotonic function. This type of behavior is usually present in polyimides at ambient temperature and for stress bigger than $1 \mathrm{MPa}$ [14] and is conventionally known as creep. Surprisingly, creep affects the proposed structure and creep-caused membrane deflections of some $\mu \mathrm{m}$ should be expected, manifesting themselves in time-scales of tens of minutes. First of all, observe that the mechanical deformation of a body subject to creep phenomena is a function of the entire loading history of the body itself. In other terms, thanks to viscoelasticity the system gains memory: each loading step, appended in the past, contributes to the final response. The Boltzmann superposition principle is a useful means of analyzing the creep deformation resulting from several distinct loading or unloading steps of strain or stress [15]. The above observation implies that in order to know the exact response of a structure subject to creep, a model of its excitation should be available, describing the evolution in time of input stress (or strain). This is normally not possible in fluid dynamic applications where the input loading and its dynamic is unknown. In this case, the best that can be achieved is a bound on the maximal deviation that creep may introduce with regard to static models such as those in Section 3.1. Such bound can be roughly interpreted as an uncertainty that should be taken into account when using the sensor as a measurement device in a dynamic environment. A convenient way to obtain such bound consists in realizing that creep can be approximately classified as a low-pass phenomenon, so that a typical experiment to estimate its extent consists in applying, at $t=0$, a step-like excitation in stress spanning the whole allowable stress range and in evaluating the difference between the response at $t=0^{+}$and the response at $t \rightarrow \infty$. Intuitively, any structure subject to a slowly varying load will deform deviating from a non-viscoelastic response by no more than such quantity (the slower the load dynamics, the lower the deviation). The major reason to practice this kind of analysis is to understand how actions on the geometry and materials employed in the sensor fabrication can reduce the extent of the viscoelastic response and thus tightening the error bounds. In the modeling of creep [16], one should consider that by taking into account the viscoelastic phenomena, the deformation model of a membrane changes from a static, non-linear, time-invariant model to a dynamic, non-linear, time-invariant model. In other terms, one could in principle model the viscoelastic behavior by introducing time derivatives into the system of partial equations that rule the membrane deforma- tion. In practice, it is generally convenient not to do so. In many conditions it is handier to model creep by using equations where time-varying parameters take care of describing the dynamical effects. A particularly effective way of doing so is by the introduction of a time dependent modulus of elasticity, obtained starting from Kapton ${ }^{\circledR}$ data sheets [14]. As shown in Fig. 6a, from strain versus time curves, given by different applied stresses, the corresponding time dependent modulus of elasticity have been calculated, interpolating the strain curves, as:

$E_{i}(t)=\frac{\sigma_{i}}{\epsilon_{i}(t)}$,

where $\epsilon_{i}(t)$ is the time dependent strain, $\sigma_{i}$ is the corresponding stress and the index $i$ represents different values of stresses and temperature conditions. The approach is convenient because it leads to equation sets which fit more easily into conventional FEM simulation framework than models with explicit time derivatives. In other terms it allows creep to be simulated by a sequence of static simulations referring to different time instants. These curves (Fig. 6b) have been fitted minimizing the root mean square difference
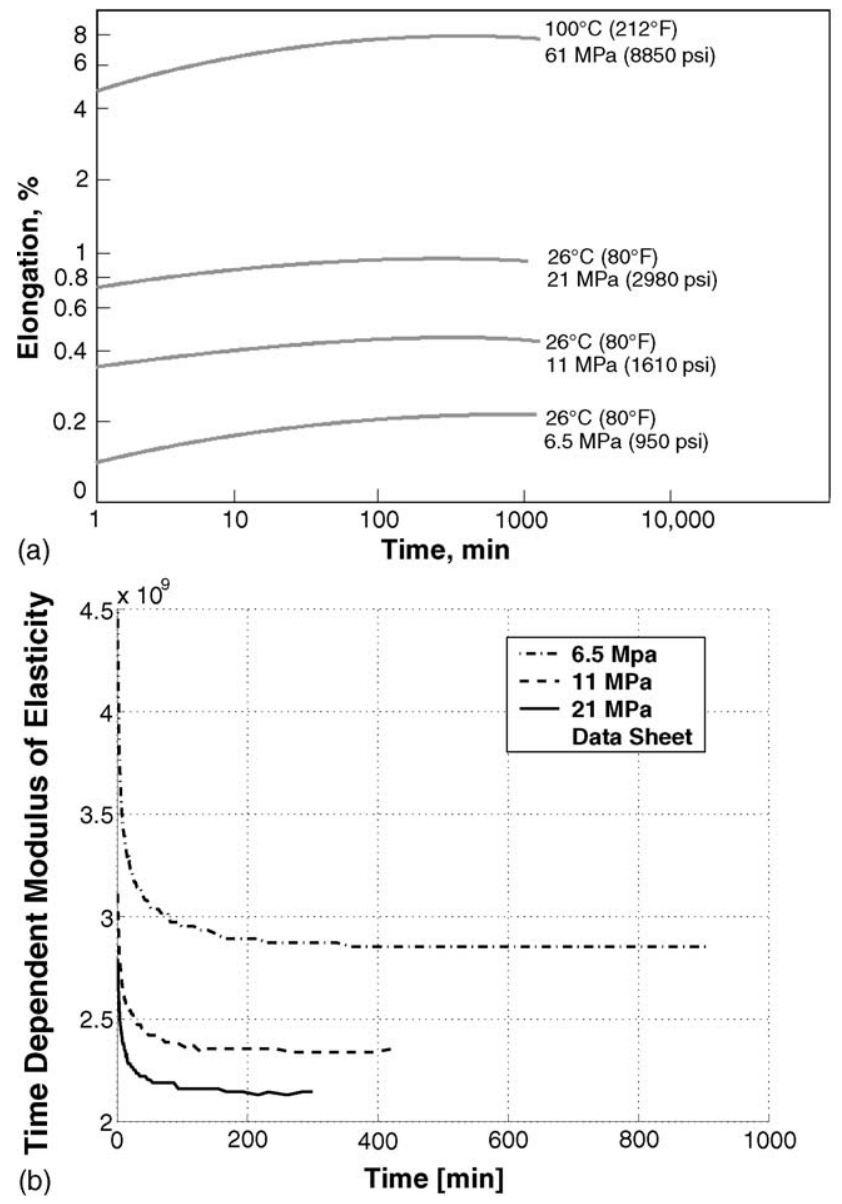

Fig. 6. Kapton creep: (a) strain versus time Kapton ${ }^{\circledR}$ creep behavior from datasheet for particular temperature and stress conditions, (b) time dependent modulus of elasticity $E(t)$, as described in Section 3.3 Eq. (7) for particular temperature and stress conditions. 
between Eq. (7) and sum of exponential and a constant, truncating the series at the fourth term:

$$
E_{i}(t)=\sum_{j}\left(A_{i_{j}} \exp B_{i_{j}} t\right)+k_{i}
$$

where $A_{i_{j}}, B_{i_{j}}$ and $K_{i}$ parameters are representative of the elastic and viscous behavior of the membrane in particular condition of exerted stress and temperature. The expressions obtained in Eq. (8) have been used for time dependent mechanical simulations. The approach followed in this work is an alternative and easier way to reproduce creep behavior without differential equation, basing the estimation of coefficients of Eq. (8) by means of interpolation, depending upon the stress and the temperature. Since the maximum membrane relaxation, due to creep, is obtained for the maximum pressure value, a time dependent capacitance variation has been calculated until the transient response can be considered finished. The goal is to predict the viscoelastic behavior in order to fix the sensor response. In fact, once the time dependent and the static behaviors have been accurately modeled, sensor design allows the sensitivity and accuracy to be defined for the targeted application.

\section{Experimental setup and results}

Experiments have been performed for testing the simulations accuracy on the sensor prototypes. Two types of analysis have been set up. A first one to reproduce and to measure the creep behavior and a second one to obtain the static characteristic of the sensors. The first experiment has been performed by applying a step of pressure of $300 \mathrm{~Pa}$ over the membranes, obtaining a capacitance variation, measured with an Agilent 4284A Precision LCR meter. As represented in Fig. 7, it is possible to distinguish two superimposed dynamics: an instantaneous response due to the elasticity of the membrane and a slower one due to the viscoelastic behavior. In order to simulate the latter phenomenon, a time dependent modulus of the elasticity has been used, as described in Section 3.2. With this approach any experimental testing or feedback analytical procedures is needed to find out FEM model parameters: any coefficient can be extracted directly from material data sheets with a good approximation. For this test, in order to apply several constant pressure values on the strip membranes, a chamber with different cavities has been used. As shown in Fig. 8, the simulation fits to the experimental viscoelastic behavior with a $1 \%$ error. For determining the static sensor characteristic, the creep behavior has been ignored in the FEM simulations. A set of different constant pressure values have been exerted on the sensor membrane, being each value applied for about $60 \mathrm{~s}$. The experimental characteristic has been obtained and compared with static simulations, as represented in Fig. 9. In order to test the performance of the strip and its functionality, a modular decomposable PVC rectangular wing based on NACA 0015 profile has been built, as a first aerodynamic test to be analyzed. Experiments have
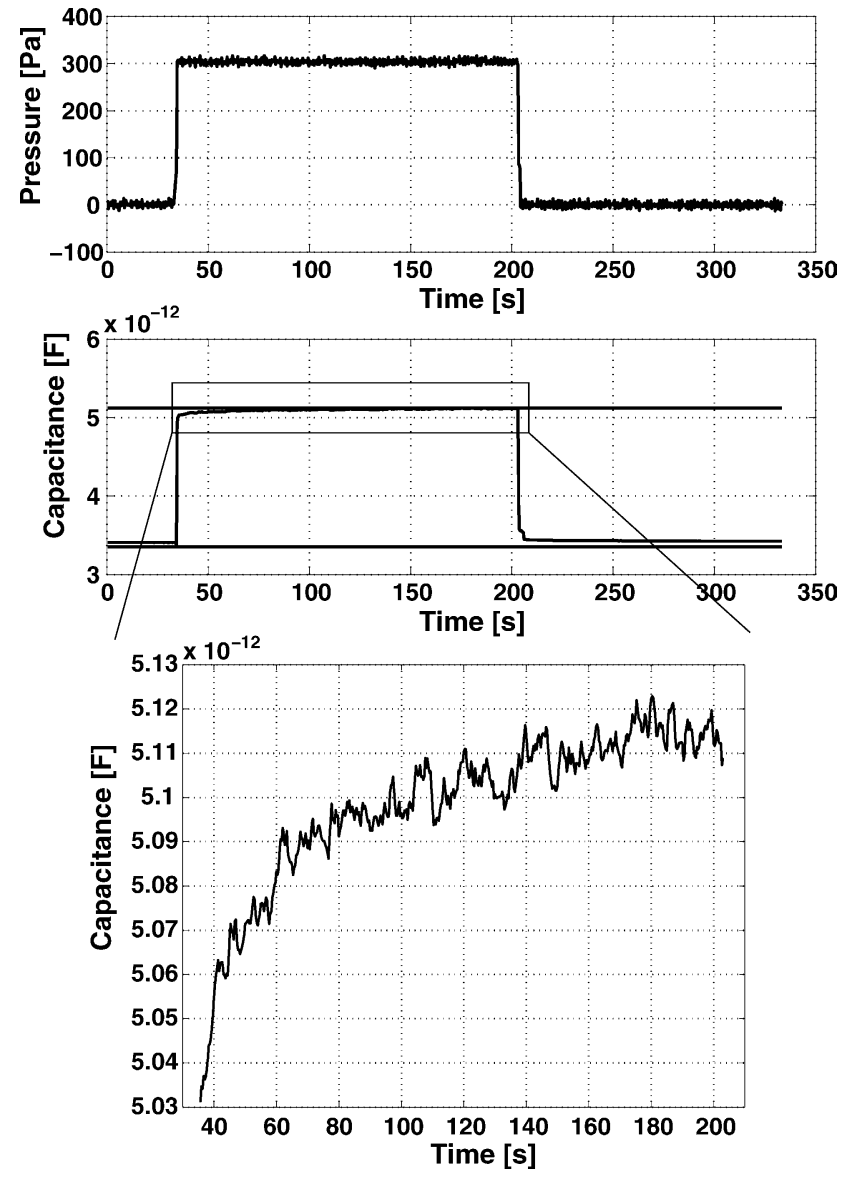

Fig. 7. Membrane capacitive response to an applied pressure step of $300 \mathrm{~Pa}$. Two superimposed dynamic are distinguished: one, faster, representative of the elastic behavior of the membrane and one representative of the viscoelastic behavior of the membrane (enlarged in the bottom). Test is relative to prototype with a $510 \mu \mathrm{m}$ spacing layer thickness.

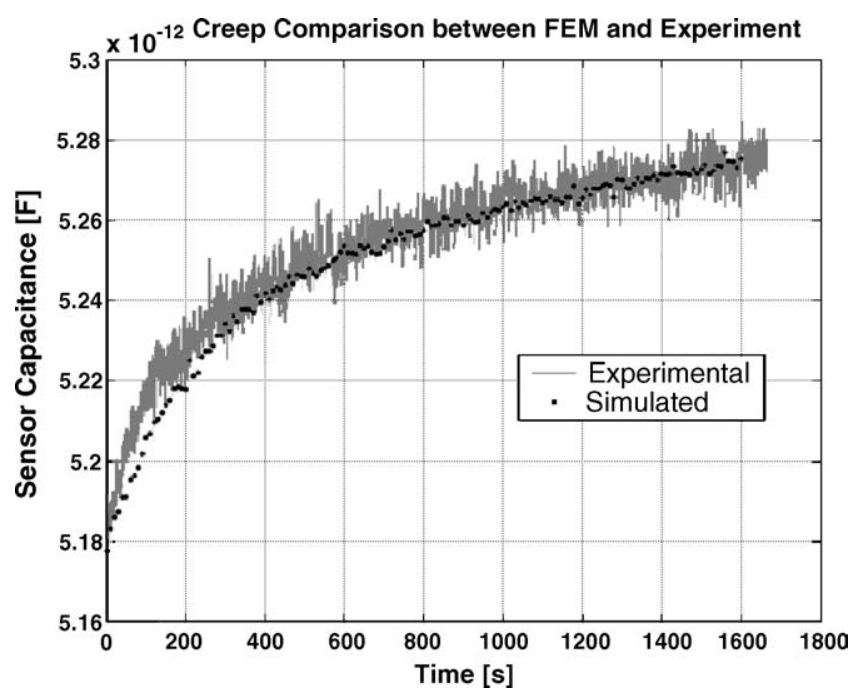

Fig. 8. Capacitive response due to membrane viscous behavior: comparison between experiment and simulation. 


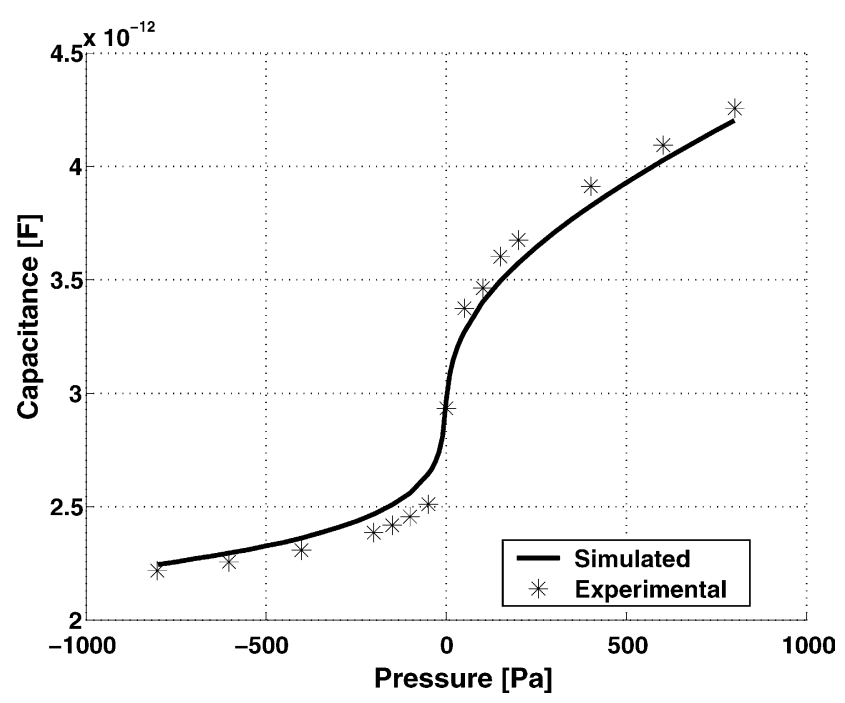

Fig. 9. Sensor characteristic curve. Data are relative to prototype with a $770 \mu \mathrm{m}$ spacing layer thickness.

been made in the wind tunnel for different angle of attack in a $\pm 10^{\circ}$ range and for different free stream velocities up to $45 \mathrm{~m} / \mathrm{s}$. The sensors strip is positioned on wing profile surfaces (Fig. 10) and data can either be acquired by a readout circuit that can be placed inside the profile, or by an Agilent 4284A Precision LCR meter providing direct measures of capacitance variation. To compare the polyimide sensors measurements with respect to a conventional technique, an orifice array has been drilled on the profile along the sensor strip and pneumatically linked to a Scanivalve ${ }^{\circledR}$ differential pressure transducer. The advantage in using this type of aerodynamic body lies in the fact that, at small angle of attack, the

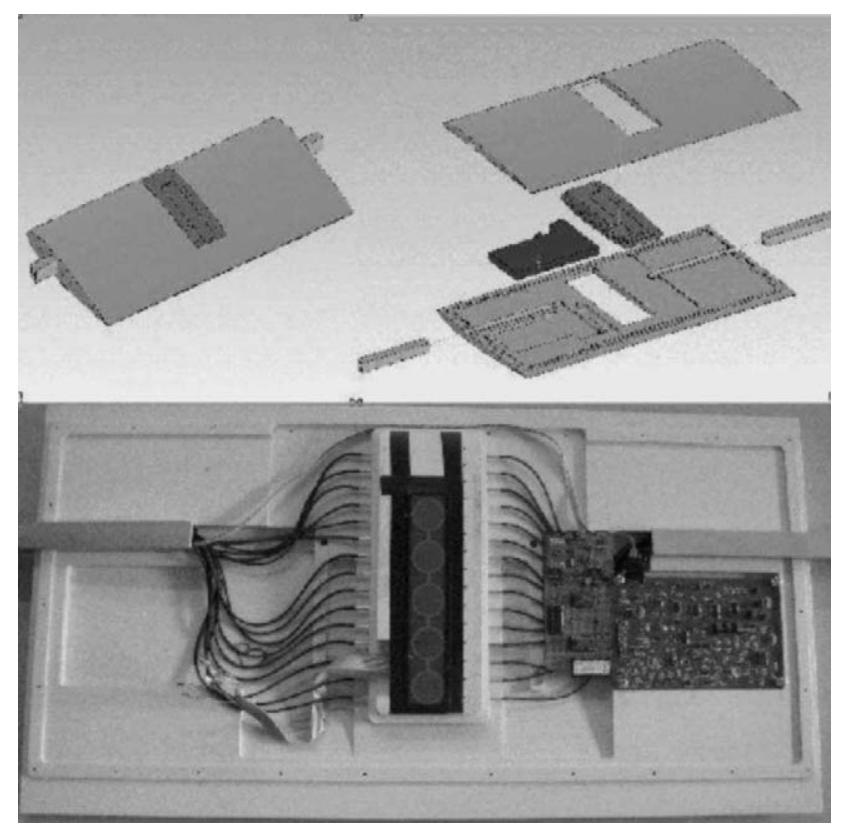

Fig. 10. Decomposable wing profile (top) and test-board for the electronic read-out circuitry (bottom).

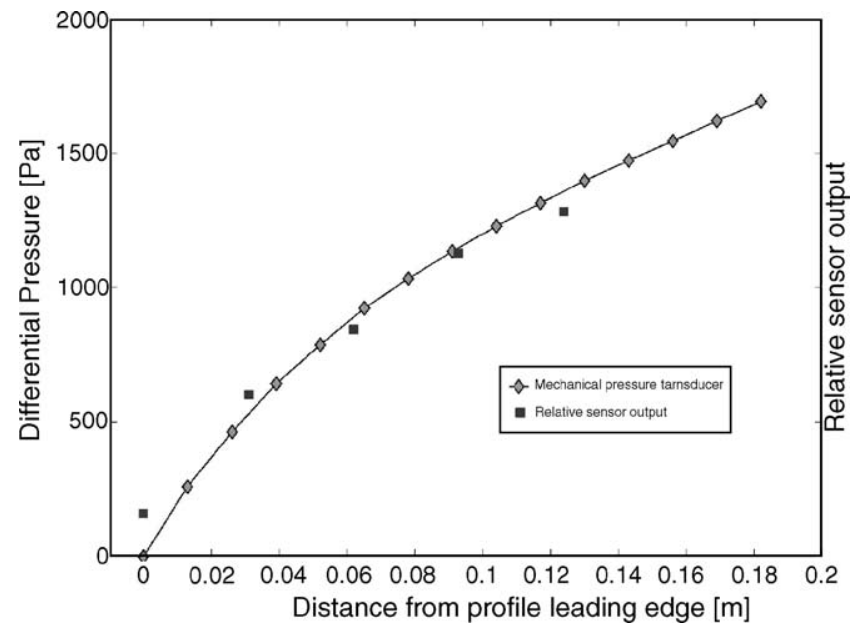

Fig. 11. Pressure distribution over the wing profile: comparison between conventional pressure transducer and the propose architecture.

results coming from the two experimental techniques can be compared and validated with the results of numerical simulations. Experimental wing profile pressure distribution data have been acquired from both the proposed and conventional sensors, as reported in Fig. 11.

\section{Discussion}

As shown in Figs. 8 and 9, experimental results have shown very good agreements with FEM simulations of pressurecapacitance static function and creep behavior. As expected, the experimental results, as forecasted by simulations, have shown a large dynamic range of electrical capacitance, in the range of $2 \mathrm{pF}$, however, creep phenomenon could decrease sensitivity for large time scales. For example, the device under test has shown creep dependent variations of about $100 \mathrm{fF}$ resulting in a $10 \%$ of degradation of dynamic scale in $20 \mathrm{~min}$ of time scale for a pressure of $300 \mathrm{~Pa}$. However, for shorter time scales, of about $1 \mathrm{~min}$, the dynamic range degradation is about $2 \%$. This problem points out the strong need of multiphysics simulations of the device: this approach greatly helps in finding the best trade-offs of the geometry before fabrication thus saving time and money. Two kinds of devices have been fabricated and placed under test, using different thickness of the spacing layers. They have shown different sensitivity and characteristic shapes, showing sensitivity from 0.5 up to $2.5 \mathrm{fF} / \mathrm{Pa}$ for the thicker sample ( $770 \mu \mathrm{m}$ of spacing layer) and from 0.67 up to $5 \mathrm{fF} / \mathrm{Pa}$ for the thinner one $(510 \mu \mathrm{m}$ of spacing layer) in $\mathrm{a} \pm 2000 \mathrm{~Pa}$ range. As pointed out before, the sensor accuracy, neglecting the electronic readout, depends upon the viscoelastic behavior that can be estimated in a range of 20-200 fF of variation depending upon the time of application and upon the pressure exerted. Device under test characteristics would result in a worst case accuracy degradation of $\pm 60 \mathrm{~Pa}$, resulting in an error of about $10 \%$ at room temperature. From the experimental results of the devices accuracy 
within this work and starting from the sensitivity and the accuracy required by the targeted application, different parameters can be trimmed for achieving best performance. First, the geometry of the membranes can be modified to achieve maximum deflection. Then, the spacing layer thickness can be changed to increase the sensitivity by way of the non linear capacitance relationship and taking into account viscoelastic degradation. Finally, the membrane thickness and materials can be chosen for reducing the time dependent effects for the targeted accuracy. As a result, the sensitivity that can be achieved with polyimide pressure sensors is comparable with that related to MEMS $[17,18]$ at lower costs in spite of the spatial resolution and pressure dynamic range.

\section{Conclusions}

The paper has introduced polymer based non-invasive sensor strip designed for acquiring pressure profiles over surfaces of aerodynamic bodies. The physical design has been assisted by multiphysic FEM simulations to better trim mode and range of operation to the application. Then, time-dependent effects of polymers has been taken into account in simulations to forecast reduction of overall sensitivity.

Two kind of experimental results have been discussed and compared to multiphysics simulations and state-of-the-art sensors performance, respectively:

(1) Static and time-dependent capacitance versus pressure function. The experimental results have shown very good agreement with simulations thus introducing a design methodology very useful for coping with trade-offs between dynamic range and sensitivity;

(2) Pressure profile acquisition. Profiles of the sensor strip have been compared with mechanical pressure acquisition systems over a typical aerodynamic body.

These results have shown how FEM-assisted multiphysics simulations help the design of cheap and flexible devices aimed at specific aerodynamic and fluid dynamic applications achieving features similar to the MEMS.

\section{References}

[1] R.J. Goldstein, Fluid Mechanics Measurements, Springer Verlag, 1983 for a classical review.

[2] R.E. Oosterbroek, T.S.J. Lammerink, J.W. Berenschot, A. Van den Berg, M.C. Elwenspoek, Designing, realization and characterization of a novel capacitive pressure/flow sensor, Solid State Sens. Actuators, 1997, 1997 International Conference on TRANSDUCERS 97 Chicago, vol. 1, 16-19 June 1997, pp. 151-154.

[3] S.T. Cho, K. Najafi, C.L. Lowman, K.D. Wise, An ultrasensitive silicon pressure-based flowmeter, Electron Devices IEEE Trans. 39 (4) (1992) $825-835$.

[4] O. Berberig, K. Nottmeyer, J. Mizuno, Y. Kanai, T. Kobayashi, The Prandtl micro flow sensor (PMFS): a novel silicon diaphragm capacitive sensor for flow-velocity measurement, Sens. Actuators A: Phys. 66 (1-3) (1998) 93-98.
[5] G.M.J. Ander, W.N. MacPherson, J.S. Barton, R.L. Reuben, J.D.C. Jones, R. Stevens, K.S. Chana, S.J. Anderson, T.V. Jones, Embedded micromachined fiber-optic Fabry-Perot pressure sensors in aerodynamics applications, Sens. J. IEEE 3 (1) (2003) 102-107.

[6] K. Asai, K. Nakakita, M. Kameda, K. Teduka, Recent topics in fastresponding pressure-sensitive paint technology at National Aerospace Laboratory, Instrumentation in Aerospace Simulation Facilities, 19th International Congress on ICIASF 2001, pp. 25-36.

[7] T. Shimoto, K. Matsui, Y. Shimada, K. Utsumi, New high-density multilayer technology on PCB, IEEE Trans. Adv. Pack. 22 (1999) 2.

[8] T. Merkel, M. Graeber, L. Pagel, A new technology for fluidic Microsystems based on PCB technology, Sensors and Actuators A 77 (1999) 98-105.

[9] S.P. Timoshenko, S. Woinowsky-Krieger, Theory of plates and shells, second ed., McGraw-Hill International Editions, 1959, Chapter 13.

[10] H. Hencky, Z. Math, Uber den Spannungszustand in kreisrunden Platten, Z. Math. Phys. 63 (1915) 311-317.

[11] http://www.comsol.com/.

[12] A. Becker, Background to Finite Element Analysis of Geometric Nonlinearity Benchmarks, Benchmark Test, NAFEMS, 2000. Sec. 5.2.

[13] N.G. Mc Crum, C.P. Buckley, C.B. Bucknall, Principles of Polymer Engineering, second ed., Oxford University Press, Oxford, 2000.

[14] DuPont Kapton DataSheets, downloaded at http://www.dupont. com/kapton/.

[15] Encyclopedia of Polimer Science and Engineering, vol. 9, Mechanical Properties, p. 390, Copyright@ 1987. by John Wiley and Sons, Inc.

[16] V.E. Smirnova, N.A. Dolotova, V.I. Aleshin, V.P. Volodin, Study of creep in polyimide film, Polym. Sci., Ser. A 40 (7) (1998) 730-735.

[17] Y. Zhang, S. Massoud-Ansari, G. Meng, W. Kim, N. Najafi, An ultra-sensitive, high-vacuum absolute capacitive pressure sensor, Micro Electro Mech. Syst., 2001, The 14th IEEE International Conference on MEMS 2001, 21-25 January 2001, pp. 166-169.

[18] S.T. Moe, K. Schjølberg-Henriksen, D.T. Wang, E. Lund, J. Nysæther, L. Furuberg, M. Visser, T. Fallet, R.W. Bernstein, Capacitive differential pressure sensor for harsh environment, Sens. Actuators A: Phys. $83(1-3)(2000)$ 30-33.

\section{Biographies}

Michele Zagnoni received the Dr Eng degree in electronic engineering from the University of Bologna, Bologna, Italy, in 2002. At the end of 2002 he joined the Advanced Research Center for Electronic Systems for Information and Communication Technologies "E. De Castro" (ARCES), Bologna, Italy. $\mathrm{He}$ is currently $\mathrm{PhD}$ student in electronic engineering at the Department of Electronics and Computer Science (DEIS) of the same university. His current research interests and activities include sensor design, pressure sensors, finite element method simulations, impedance analysis of biological matter.

\footnotetext{
Alessandro Golfarelli received the degree in electronic engineering from the University of Bologna, Italy, Bologna, in 1999. From 2000 to 2002, he was with the Laboratori Fondazione G. Marconi of Pontecchio Marconi, Bologna, Italy, working as integrated circuit designer in MMIC and RFIC area. He is currently a collaborator with the Advanced Research Center on Electronic System "E. DeCastro" (ARCES), Bologna, Italy. His research focuses on acquisition systems for sensor network.
}

Sergio Callegari received the Dr Eng degree (with honors) in electronic engineering and the $\mathrm{PhD}$ degree in electronic engineering and computer science from the University of Bologna, Bologna, Italy, in 1996 and 2000, respectively, working on the study of nonlinear circuits and chaotic systems. In 1996, he was a visiting student at King's College London, London, UK. He is currently a researcher at the II School of Engineering, University of Bologna, where he is a lecturer in digital electronics and sensors. At the same University he has also joined the recently instituted 
Advanced Research Center on Electronic Systems for Information and Communication Technologies. His current research interests include sensor design, nonlinear signal processing, internally nonlinear, externally linear networks, and chaotic maps. He has authored or co-authored more than 35 papers in international conferences, journals and scientific books. In 2004 he was recipient of the IEEE Circuit and Systems Society Darlington Award.

Virna Bonora received her degree in 1982 in industrial chemistry at the University of Bologna. Her research interest started with the synthesis and characterization of polymer materials supported by grant of AIRI and Accademia Nazionale dei Lincei. In 1989 received the PhD degree in industrial chemistry, at the same University, with a dissertation on synthesis and characterization of block copolymers as "compatibilizer" in polymer blends. She spent some time at the Donnan Laboratories of the University of Liverpool. In 1992 she joined the University of Bologna as assistant professor at the Department of Applied Chemistry and Materials Science where she is working and teaching in the field of materials science.

Alessandro Talemelli graduated in aeronautical engineering (with honors) at the University of Pisa in 1990. He became a PhD in Aerospace Engineering at Politecnico di Milano, 1994. Researcher in fluid mechanics at Università of Pisa from 1995, he became associate professor in aerodynamics at the University of Bologna in 2001. Since 2003 he is guest lecturer at the Royal Institute of Technology KTH in Stockholm and Associate Lecturer at the University of Modena. He does his main activity in fluid mechanics, in particular experimental studies of turbulence in jets and confined flows. He is author in more than 40 papers in journals and conferences.

Enrico Sangiorgi was born in Faenza, Italy, in 1954. He received the Laurea degree in electrical engineering from the University of Bologna, Italy, in 1979. In 1993, he was appointed full professor of electronics at the University of Udine, Italy, where he started the Electrical Engineering Program and the Microelectronic Group. In 2002, he joined the University of Bologna, where he is head of the microelectronics group in the satellite Campus of
Cesena-Forli (II School of Engineering). From 1985 to 2001, he was a consultant at Bell Labs-Lucent Technologies, Murray Hill, NJ, where he was a resident visitor for more than three years. In 1983, 1984, and 1991, he was a visiting scientist at the Center for Integrated Systems, Stanford University, Stanford, CA. His research interests include the physics, characterization, modeling, and fabrication of silicon solid-state devices and integrated circuits. In particular, he has been working on several aspects of device scaling, its technological, physical, and functional limits, as well as device reliability for silicon MOS and bipolar transistors. In order to tackle and eventually overcome the hurdles of device scaling, he has devised and developed several original concepts and methods in the characterization and modeling of nanoscale silicon devices. He has co-authored 28 IEDM papers and more than 140 papers published in international journals and conference proceedings. Dr. Sangiorgi is a Member of the IEEE Electron Devices Society (EDS) TCAD Committee and the EDS Regions/Chapter Committee. Since 1994, he has been an editor for the IEEE ELECTRON DEVICE LETTERS. He has been a member of the technical committees of IEDM, ESSDERC, ULIS, and INFOS.

Marco Tartagni received the Laurea and the $\mathrm{PhD}$ degree in electrical engineering and computer sciences from the University of Bologna, Italy, in 1988 and 1993, respectively. He joined the Department of Electrical Engineering, California Institute of Technology, Pasadena, in 1992 as a visiting student and in 1994 as research fellow, working on various aspects of analog VLSI for imaging processing. Since 1995, he has been with the Department of Electronics, University of Bologna, where he is currently associate professor of Electrical Engineering, working on optical and capacitive CMOS smart sensors. From 1996 to 2002 he was with the STMicroelectronics and University of Bologna joint lab as team leader developing innovative capacitive fingerprint sensors for biometrics. In 2002 he joined the Center of Excellence on Electronics Systems ARCES of the same University focusing his research on integrated microsystems, analog design, sensor interfaces and hybrid technologies for cellular and molecular biology. He is co-founder of Silicon Biosystems s.r.l. and co-recipient of the ISSCC 2004 Van Vessem Outstanding Paper Award. 